\title{
Regeneration of Drosophila sensory neuron axons and dendrites is regulated by the Akt pathway involving Pten and microRNA bantam
}

\author{
Yuanquan Song, Kassandra M. Ori-McKenney, Yi Zheng, Chun Han, Lily Yeh Jan, and Yuh Nung Jan ${ }^{1}$ \\ Howard Hughes Medical Institute, Department of Physiology, Department of Biochemistry and Biophysics, University of \\ California at San Francisco, San Francisco, California 94158, USA
}

\begin{abstract}
Both cell-intrinsic and extrinsic pathways govern axon regeneration, but only a limited number of factors have been identified and it is not clear to what extent axon regeneration is evolutionarily conserved. Whether dendrites also regenerate is unknown. Here we report that, like the axons of mammalian sensory neurons, the axons of certain Drosophila dendritic arborization (da) neurons are capable of substantial regeneration in the periphery but not in the CNS, and activating the Akt pathway enhances axon regeneration in the CNS. Moreover, those da neurons capable of axon regeneration also display dendrite regeneration, which is cell type-specific, developmentally regulated, and associated with microtubule polarity reversal. Dendrite regeneration is restrained via inhibition of the Akt pathway in da neurons by the epithelial cell-derived microRNA bantam but is facilitated by cell-autonomous activation of the Akt pathway. Our study begins to reveal mechanisms for dendrite regeneration, which depends on both extrinsic and intrinsic factors, including the PTEN-Akt pathway that is also important for axon regeneration. We thus established an important new model system-the fly da neuron regeneration model that resembles the mammalian injury model-with which to study and gain novel insights into the regeneration machinery.
\end{abstract}

[Keywords: CNS; Pten; bantam; dendritic arborization neuron; microRNA; regeneration]

Supplemental material is available for this article.

Received March 30, 2012; revised version accepted May 29, 2012.

Damage to the adult CNS often leads to persistent deficits due to the inability of axons to regenerate after injury, which may reflect an inhibitory extrinsic environment and a diminished intrinsic regenerative capacity (Schwab and Bartholdi 1996; Goldberg et al. 2002; Filbin 2006; Fitch and Silver 2008). Whereas CNS axon regeneration may be impeded by inhibitory molecules associated with the myelin debris (e.g., MAG, Nogo-A, and OMgp) or with glial scar formation (e.g., CSPG and tenasin) (Yiu and He 2006; Sun and He 2010), eliminating these molecules only allows limited sprouting in vivo (Case and TessierLavigne 2005; Harel and Strittmatter 2006; Yiu and $\mathrm{He}$ 2006; Sun and He 2010). Whether axon regeneration could succeed also depends on the intrinsic competence of injured neurons in launching a growth program (Sun and $\mathrm{He}$ 2010). To date, studies using different models have collectively implicated the involvement of transcription factors

\footnotetext{
${ }^{1}$ Corresponding author

E-mail yuhnung.jan@ucsf.edu

Article published online ahead of print. Article and publication date are online at http://www.genesdev.org/cgi/doi/10.1101/gad.193243.112.
}

(Moore et al. 2009), signaling pathways (Park et al. 2008; Hammarlund et al. 2009; Yan et al. 2009), and microRNAs (miRNAs) (Williams et al. 2009). Recent genetic screens in worms have uncovered additional regeneration factors that affect axon guidance, membrane excitability, neurotransmission, and synaptic vesicle endocytosis (Chen et al. 2011). However, our current understanding remains incomplete (Sun and He 2010). Additional insights may come from examining the extent of evolutionary conservation of mechanisms underlying axon regeneration in mammals and genetically tractable organisms such as the fruit fly.

Compared with axon regeneration, much less is known about dendrite regeneration. We chose to explore questions concerning axon and dendrite regeneration in Drosophila melanogaster, which has been instrumental in defining genes and signaling pathways that are evolutionarily conserved in eukaryotes, including humans, thereby providing valuable insights into the studies of human diseases, including neurodegeneration (Marsh and Thompson 2006; Lessing and Bonini 2009; Lu and Vogel 2009). We focused our study on Drosophila dendritic arborization 
(da) neurons, a type of sensory neuron used extensively to study dendrite morphogenesis and remodeling /Gao et al. 1999; Grueber et al. 2002; Sugimura et al. 2003; Kuo et al. 2005, 2006; Williams and Truman 2005; Williams et al. 2006; Parrish et al. 2007), because they provide an opportunity to test dendrite regeneration and compare the extent of axon regeneration in the periphery versus the CNS. Drosophila da neurons can be subdivided into four classes based on dendritic branching morphology: class I to class IV (Grueber et al. 2002). With the cell body of these sensory neurons in the periphery and their elaborate dendritic branches in the body wall, each da neuron sends out an axon that travels through peripheral tissues and forms synaptic connections in the CNS, the ventral nerve cord (VNC), similar to the way vertebrate sensory neurons of the dorsal root ganglion (DRG) project into the spinal cord. DRG neuronsa widely used model for spinal cord injury-have peripheral processes capable of regeneration, whereas their central axons that project into the spinal cord fail to regrow after injury (Ramon y Cajal 1928). In this study, we show that da neurons exhibit axon regeneration in the periphery but not in the CNS, thereby exhibiting features similar to that of DRG neurons. We further demonstrate dendrite regeneration of da neurons and identify cell-autonomous as well as nonautonomous factors important for axon/ dendrite regeneration.

The PTEN/mTOR pathway has been implicated as a critical neuronal intrinsic regulator of axon regeneration. Neuronal PTEN deletion in mice enhances axon regeneration of retinal ganglion cells after optic nerve injury (Park et al. 2008) as well as axon regeneration of corticospinal neurons after spinal cord lesion (Liu et al. 2010). In this study, we provide evidence that PTEN and its downstream target, Akt, are important not only for axon regeneration in the CNS, but also for dendrite regeneration of Drosophila da neurons.

The finding of muscle-specific miRNA-206 in promoting regeneration of neuromuscular synapses in mice (Williams et al. 2009) raises the question of whether miRNA may be involved in axon/dendrite regeneration. To approach this question, we examined the role of the miRNA bantam (ban), which is known to function in epithelial cells to regulate dendrite scaling by dampening Akt activity in adjacent da neurons (Parrish et al. 2009), and found that bantam also regulates dendrite regeneration.

In summary, we show here that class IV da neurons can regenerate their axons in the periphery but not inside the CNS and that activating the Akt pathway promotes regeneration in the CNS. This is the first report of such a case in invertebrates and underscores the evolutionary conservation of mechanisms underlying axon regeneration. Whereas dendrite regeneration has not been clearly demonstrated previously, we show here that da neuron dendrites are capable of regeneration, but with class specificity and temporal regulation. Our study further implicates the involvement of the Akt pathway acting together with miRNA in its regulation.

\section{Results}

Regeneration of da neuron axons in the periphery depends on the neuronal type and the presence of glial processes

To determine whether da neuron axons in the periphery are capable of regeneration, we first analyzed the class IV da neurons ddaC and v'ada, whose dendrites display complex morphology and tile the entire larval body wall. We used pickpocket-CD4-tdGFP (ppk-CD4-tdGFP) to label class IV da neurons specifically. We performed axotomy of second instar larvae $48 \mathrm{~h}$ after egg laying (AEL) and targeted the lesion to the axon segment $\sim 20-50 \mu \mathrm{m}$ from the cell body (Supplemental Fig. S1A). After $24 \mathrm{~h} \mathrm{(72} \mathrm{h}$ AEL), the same neuron was imaged to confirm the complete transection; by then, the distal part of the severed axon had largely degenerated. Two days later (120 h AEL, right before puparium formation), the same neuron was imaged again to assess regeneration. We found these class IV da neuron axons are highly regenerative, with $64.3 \%$ and $74.4 \%$ of ddaC and $\mathrm{v}^{\prime}$ ada neurons, respectively, displaying regeneration (Fig. 1M). New axons originated from the severed stem (Fig. 1A,E, circle) and extended along the original axon trajectory and beyond the lesion site (Fig. 1B,F, arrowheads). "Regeneration index," which is defined as the increase of axon length normalized to the body size, thus depicting whether the severed axon regrows beyond the lesion site (see the Materials and Methods; Supplemental Fig. S1J,K), was 0.19 and 0.49 for ddaC and v'ada (Fig. 1O), and the axon length change was $47.5 \pm 13.9 \mu \mathrm{m}$ and $137.4 \pm 18.2 \mu \mathrm{m}$ (mean \pm SEM), respectively. Whenever $\mathrm{ddaC}$ and v'ada axons displayed regeneration, they always grew along glial processes, which were marked by repo-Gal4>mRFP (Fig. $1 \mathrm{~A}^{\prime \prime}, \mathrm{B}^{\prime \prime}, \mathrm{E}^{\prime \prime}, \mathrm{F}^{\prime \prime}$ ). Strikingly, the $\mathrm{v}^{\prime}$ ada neurons sometimes regrew their axons in the direction opposite to their normal trajectory, and yet the new axons still followed faithfully the glial processes (Supplemental Fig. S1B,C, arrowheads). To test whether glial processes are required for axon regeneration, we compared the extent of axon regeneration in the glia ${ }^{+}$ group, in which only axons were severed without obvious damage to the glial processes, and the glia ${ }^{-}$group, in which both axons and glial processes were severed, while the glial cell bodies were not damaged. We found that damage of glial processes led to failed axon regeneration (Fig. $1 \mathrm{C}, \mathrm{C}^{\prime \prime}, \mathrm{D}, \mathrm{D}^{\prime \prime}, \mathrm{G}, \mathrm{G}^{\prime \prime}, \mathrm{H}, \mathrm{H}^{\prime \prime}$, circles and arrows). In the glia ${ }^{+}$group, ddaC and $\mathrm{v}^{\prime}$ ada axons regenerated $100 \%$ and $97 \%$ of the time, with regeneration index values of 0.41 and 0.62 , while in the glia ${ }^{-}$group, there was no regeneration for either $\mathrm{ddaC}$ or v'ada, with regeneration index values close to 0 (Fig. $1 \mathrm{~N}, \mathrm{P}$ ). Thus, the ability of class IV da neuron axons to regenerate depends on the presence of glial processes. While our results provide a tight association of axon regeneration with the presence of glial processes, we cannot rule out the possibility that other remaining axons may also play a permissive role. In our analyses, we noticed that neurons with severed axons appeared atrophic as compared with uncut controls; however, they still showed substantial axon 


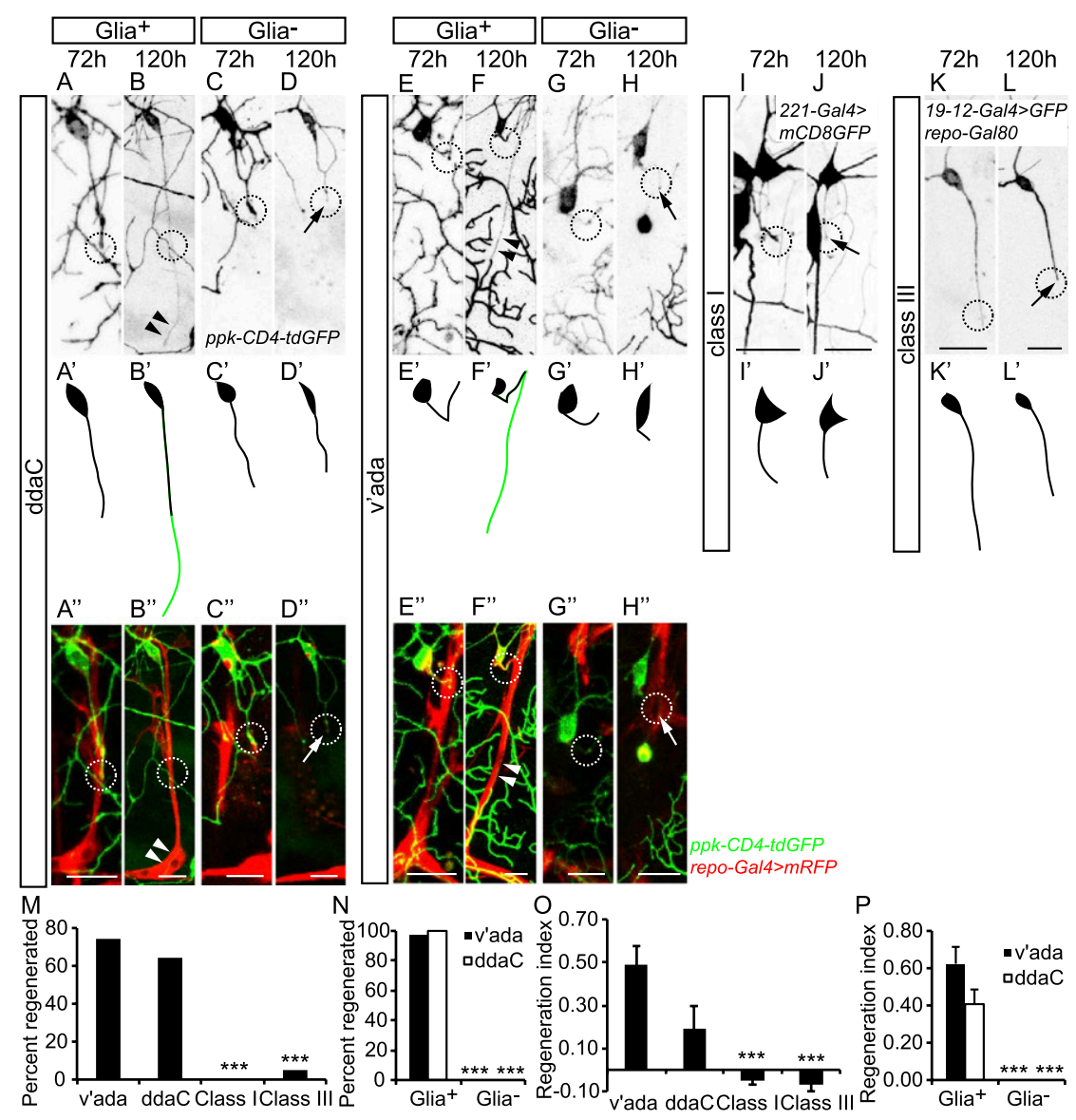

Figure 1. da neuron axon regeneration in the periphery displays class specificity and glia dependence. $(A-D)$ Axon regeneration of class IV da neuron ddaC (ppk-CD4-tdGFP/+; repo-Gal4, $U A S-m R F P /+)$. $\left(A-B^{\prime \prime}\right)$ In the glia ${ }^{+}$group, where only axons were severed (circle) without obvious damage to the wrapping glia processes, ddaC axons regrew beyond the lesion site, following a trajectory demarcated by glia processes (arrowheads). $\left(C-D^{\prime \prime}\right)$ In the glia ${ }^{-}$group, where both axon and glia processes were severed (circle), $\mathrm{ddaC}$ axons failed to show regeneration (arrow). $\left(A^{\prime}-L^{\prime}\right)$ Schematic drawings of the neurons in $A-L$, depicting only the cell body and axon. $(E-H)$ Axon regeneration of class IV da neuron v'ada. $\left(E-F^{\prime \prime}\right)$ In the glia ${ }^{+}$group, v'ada axons were also able to extend beyond the lesion site (circle) and regenerate along glia processes (arrowheads). ( $\left.G-H^{\prime \prime}\right)$ In the glia ${ }^{-}$ group, v'ada axons also failed to regenerate (arrow). $(I, J)$ Axons of class I da neurons, as labeled with 221-Gal4, UAS-mCD8-GFP, failed to regenerate beyond the lesion site (circle and arrow). $(K, L)$ Axons of class III da neurons, as labeled with repo-Gal80/+; 19-12-Gal4, UAS-CD4-tdGFP/+, failed to regenerate beyond the lesion site (circle and arrow). Bar, $20 \mu \mathrm{m}$. (M-P) Quantification of axon regeneration. $(M)$ Regeneration percentage for class I and class III da neurons is significantly lower than that for v'ada and ddaC. Fisher's exact test, $\left(^{\star \star \star}\right) P<0.0005$. $n=43,14,18$, and 19 for $\mathrm{v}^{\prime} \mathrm{ada}$, ddaC, class I, and class III. $(N)$ Regeneration percentage for $\mathrm{v}^{\prime}$ ada and ddaC in the glia ${ }^{-}$group $\left(n=10\right.$ and 5 for v'ada and ddaC) is significantly lower than that in the glia ${ }^{+}$group $\left(n=33\right.$ and 9 for $\mathrm{v}^{\prime}$ ada and ddaC). Fisher's exact test, $\left(^{\star \star \star}\right) P<0.0005$. (O) Regeneration index for class I and class III is significantly lower than that for v'ada and ddaC. One-way ANOVA followed by Dunn's test, $\left(^{\star \star \star}\right) P<0.0005 . n=36,13,18$, and 19 for v'ada, ddaC, class I, and class III. $(P)$ Regeneration index for $\mathrm{v}^{\prime}$ ada and $\mathrm{ddaC}$ in the glia ${ }^{-}$group $\left(n=7\right.$ and 5 for $\mathrm{v}^{\prime}$ ada and $\left.\mathrm{ddaC}\right)$ is significantly lower than that in the glia ${ }^{+}$ group $(n=30$ and 9 for v'ada and $\mathrm{ddaC})$. Student's $t$-test, $\left({ }^{\star \star \star}\right) P<0.0005$.

regeneration regardless of the atrophy, suggesting that axon regeneration is not directly correlated with cell body atrophy.

To ask whether all da neuron axons possess the regeneration capacity, we examined class I da neurons, which are marked with 221-Gal4>mCD8-GFP and have dendrites with relatively simple morphology encompassing a smaller dendritic field. We found that class I da neuron axons did not exhibit significant regrowth after lesion (Fig. 1I,J, circle and arrow), with $0 \%$ of the neurons exhibiting regeneration, a regeneration index of -0.05 (Fig. 1M,O), and an axon length change of $2.0 \pm 2.1 \mu \mathrm{m}$, reflecting axon stalling or retraction. Consistent with previous findings (Stone et al. 2010), we found that class I da neurons sometimes converted their dendrites into axon-like processes but displayed no de novo axon regeneration.

Last, we analyzed class III da neurons, which are marked with 19-12-Gal4>UAS-CD4-tdGFP (Xiang et al. 2010) together with repo-gal80 (Awasaki et al. 2008) to eliminate the expression in glial cells. Class III da neurons resemble class IV but not class I da neurons in their dendrite scaling and tiling (Parrish et al. 2009). However, we found that class III da neuron axons also failed to regenerate after lesion (Fig. $1 \mathrm{~K}, \mathrm{~L}$, circle and arrow), with $0.05 \%$ of the neurons exhibiting regeneration, a regeneration index of -0.07 (Fig. 1M,O), and an axon length change of $14.3 \pm$ $4.6 \mu \mathrm{m}$. Therefore, even though both class IV and class III da neurons display dendritic scaling and tiling, their axon regeneration properties differ dramatically.

To verify that axon regeneration is a cell type-specific feature, we severed the axons of both class IV and class I da neurons at the same location in the same body segment of larvae, with their class IV ddaC neurons and class I da neurons marked with ppk-CD4-tdTomato and 221-Gal4> $m C D 8-G F P$, respectively. With the complete severance of all of the axons confirmed $24 \mathrm{~h}$ after lesion (Supplemental Fig. S1D,F, circle), we found that ddaC axons but not class I da neuron axons showed extensive regeneration beyond the lesion site (Supplemental Fig. S1E,G, arrowheads and arrow). These observations indicate that class IV but not class I or class III da neurons are competent in regenerating their axons in the periphery. 
The da neuron axon regeneration capacity is similar at different stages of larval development

To ask whether da neuron axon regeneration is dependent on the stage of larval development, we performed axotomy of the class IV da neuron v'ada at a later time point, $72 \mathrm{~h} \mathrm{AEL}$, and found robust axon regeneration (Supplemental Fig. S2A,B), as evident from the percentage of neurons showing regeneration and regeneration index (the axon length change was $117.6 \pm 26.4 \mu \mathrm{m}$ ) (Supplemental Fig. S2E,F), and axons also regrew along glia processes (Supplemental Fig. S2B-B "' ${ }^{\prime}$, arrowheads). Since severing of class I da neuron axons at $48 \mathrm{~h}$ AEL showed no observable regeneration, and class I neurons establish their coverage earlier than class IV da neurons (Parrish et al. 2009), we also looked at earlier time points. No axon regeneration of class I da neurons was observed even if axotomy was performed at $24 \mathrm{~h}$ AEL (the axon length change was $13.0 \pm 4.3 \mu \mathrm{m}$ ) (Supplemental Fig. S2C,D,G,H), indicating that the ability of da neuron axons to regenerate in the periphery depends on the cell type but not the developmental stage.

\section{Axon injury leads to mixed microtubule polarity in the closest dendrites and the regenerating axon}

A critical step during regeneration is the propagation of the injury signal to the cell body and the transportation of cytoskeletal elements as well as membranes toward the injury site by molecular motors, which rely heavily on the microtubule polarity. We therefore examined microtubule polarity after axon injury using EB1-GFP, which binds to the growing microtubule plus end (Zheng et al. 2008; Stone et al. 2010). In control class IV da neurons without any lesion, EB1 comets in the dendrites move exclusively retrogradely toward the cell body (Fig. 2A), as indicated by the unidirectional lines that are in parallel in the kymograph (Fig. 2A), whereas axon lesion led to a surge of EB1 comets that moved anterogradely toward the distal end of dendrites (Fig. 2B), as indicated by the two sets of lines that cross each other in the kymograph (Fig. 2B). This mixed microtubule polarity is most prominent in the dendrites closest to the severed axon, in which $\sim 40 \%$ of the comets exhibited reversed polarity, and most of these anterogradely moving comets were seen to originate
A

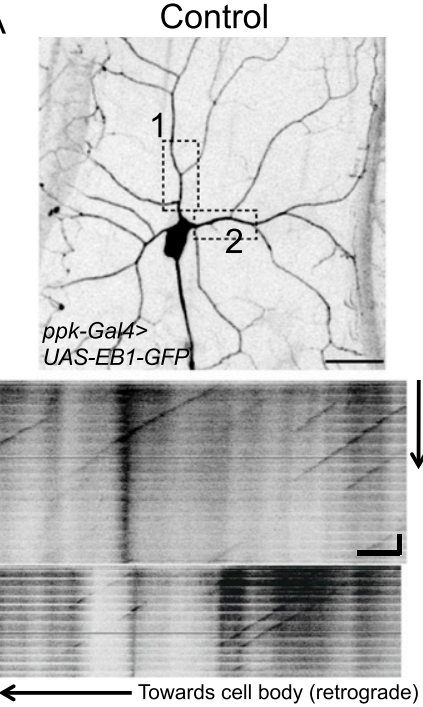

C

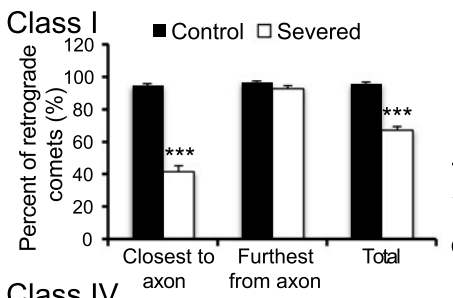

Class IV axon from axon

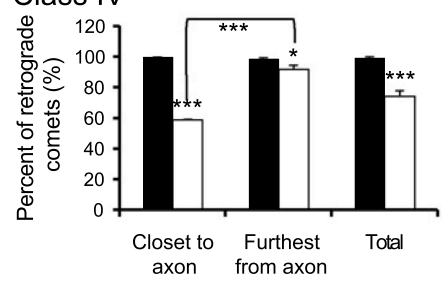

B

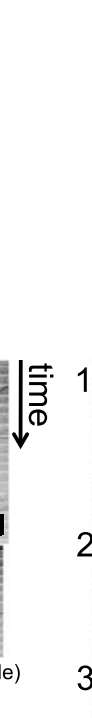

Axon severed
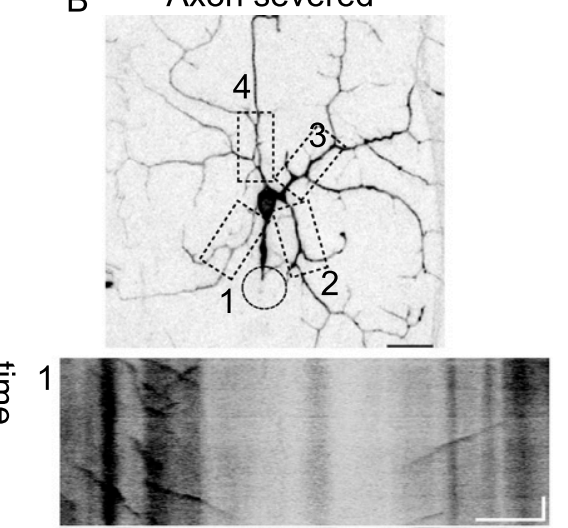

2

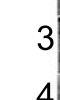

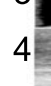
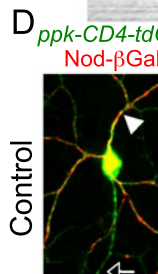
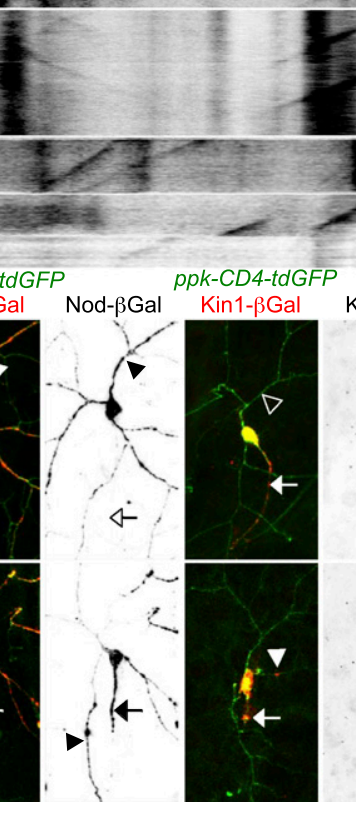

Kin1- $\beta$ Gal

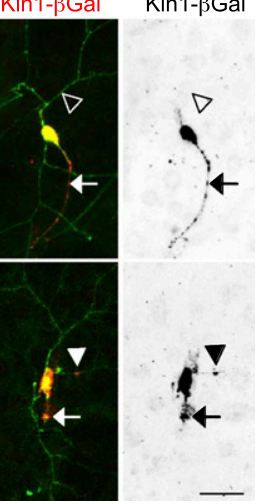

Figure 2. Axotomy of class IV da neurons induces mixed microtubule orientation in the dendrites closest to the severed axon and mislocalization of

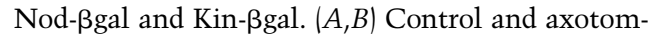
ized class IV da neurons (ppk-Gal4/UAS-EB1-GFP) and associated kymographs displaying the direction of EB1-GFP comet growth in the labeled dendrite branches. Axons were severed at $48 \mathrm{~h}$ AEL at the location represented by the dashed circle, and EB1 movies were captured at $72 \mathrm{~h} \mathrm{AEL}$ for both control and axotomized neurons. Bars: neuron images, $20 \mu \mathrm{m} ; X$-axis in kymographs, 5 $\mu \mathrm{m} ; \mathrm{Y}$-axis in kymographs, $30 \mathrm{sec}$. (C) Quantification of the percent of EB1 comets that grew toward the cell body (in the retrograde direction) in control and severed class IV and class I neurons. Student's $t$-test, $\left(^{\star}\right) P<0.05 ;\left(^{\star \star \star}\right) P<0.0005 . n>15$ neurons, and $n>150$ EB1 comets analyzed per condition. $(D)$ UAS-nod-lacZ and UAS-kin-lacZ is driven by ppkGal4. Arrows indicate proximal axons, and arrowheads indicate proximal dendrites. The localization

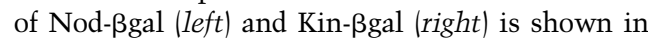
control (top panels) and severed (bottom panels) class IV ddaC neurons. In control, Nod-ßgal entered only the very proximal axon but was not present in the axon shaft. After injury, Nod-pgal extended into the axon shaft. In control, Kin- $\beta$ gal is normally localized specifically to axons. After injury, while it is still enriched within the axon, a residual amount is present in the neighboring dendritic branch. Bar, $20 \mu \mathrm{m} . n>3$ per condition. 
from the cell body (Fig. 2B,C). Interestingly, despite the inability of class I da neurons to regenerate their axons, a similar reversal of EB1 comet direction and increase of total EB1 comet number were observed (Fig. 2C; Supplemental Fig. S3A-D) as reported previously (Stone et al. 2010), suggesting that this may be a general injury response for the neuron to compensate for its loss of axon. With this mechanism in place, if the axon fails to regenerate, one or more of the neighboring dendrites are already primed to turn into an axon. Moreover, we found that, whereas in the control axon all EB1 comets are anterograde (Supplemental Fig. S3E), reflecting the uniform orientation of microtubules (Zheng et al. 2008), the regenerating axon displayed a mixed polarity (Supplemental Fig. S3F,G), raising the possibility that this may be a means for the injured axon to relay the injury and regrowth status to the cell body and thereby instruct regeneration.

To further investigate the axonal microtubule organization after injury, we then used the microtubule minus end marker Nod-ßgal (Clark et al. 1997), which localizes specifically to dendrites in da neurons (Zheng et al. 2008). Whereas in control ddaC neurons, Nod-ßgal was restricted to dendrites (Fig. 2D), in ddaC neurons in which axons were severed, Nod-ßgal was still present in dendrites but frequently localized to axons as well (Fig. 2D). Next we used the axon-specific marker Kin-ßgal /Clark et al. 1997) and found that while most of the signal remained within the axons of severed $\mathrm{ddaC}$ neurons as in the control, some signal was detected in the neighboring dendritic branch (Fig. 2D). These observations are consistent with the microtubule polarity reversal in dendrites and axons after axon lesion, as shown by EB1-GFP imaging, and raise the possibility that after axon injury, the neuron may enter a state where the intracellular trafficking and transportation are boosted and more dynamic, which could favor regeneration. Another possibility is that axon injury might result in impaired gatekeeping machinery that normally separates dendrite- versus axon-directed factors or organelles.

\section{The da neuron axons that enter the CNS are refractory to regeneration but can be coaxed to regenerate by activation of the PTEN-Akt pathway}

A salient feature of CNS damage is the failure of axons to regenerate, resulting in the breakdown of neural circuitry and irreversible neurologic dysfunction. This has been well documented in mammals; however, it is less clear whether similar phenomena take place in other organisms, apart from the observation that CNS axons from cultured adult fly brains showed limited regeneration (Ayaz et al. 2008). We therefore examined the ability of da neuron axons to regenerate within the VNC, aiming to generate an in vivo fly model comparable with the spinal cord injury model in mice.

To produce axon lesion in the CNS, we performed axotomy at $48 \mathrm{~h} \mathrm{AEL}$ by targeting the laser to an area of $\sim 20 \mu \mathrm{m}$ in diameter at the commissure junction in the VNC (Fig. 3A) of larvae expressing the class IV da neuron marker ppk-CD4-tdGFP. We then imaged at $72 \mathrm{~h}$ AEL to

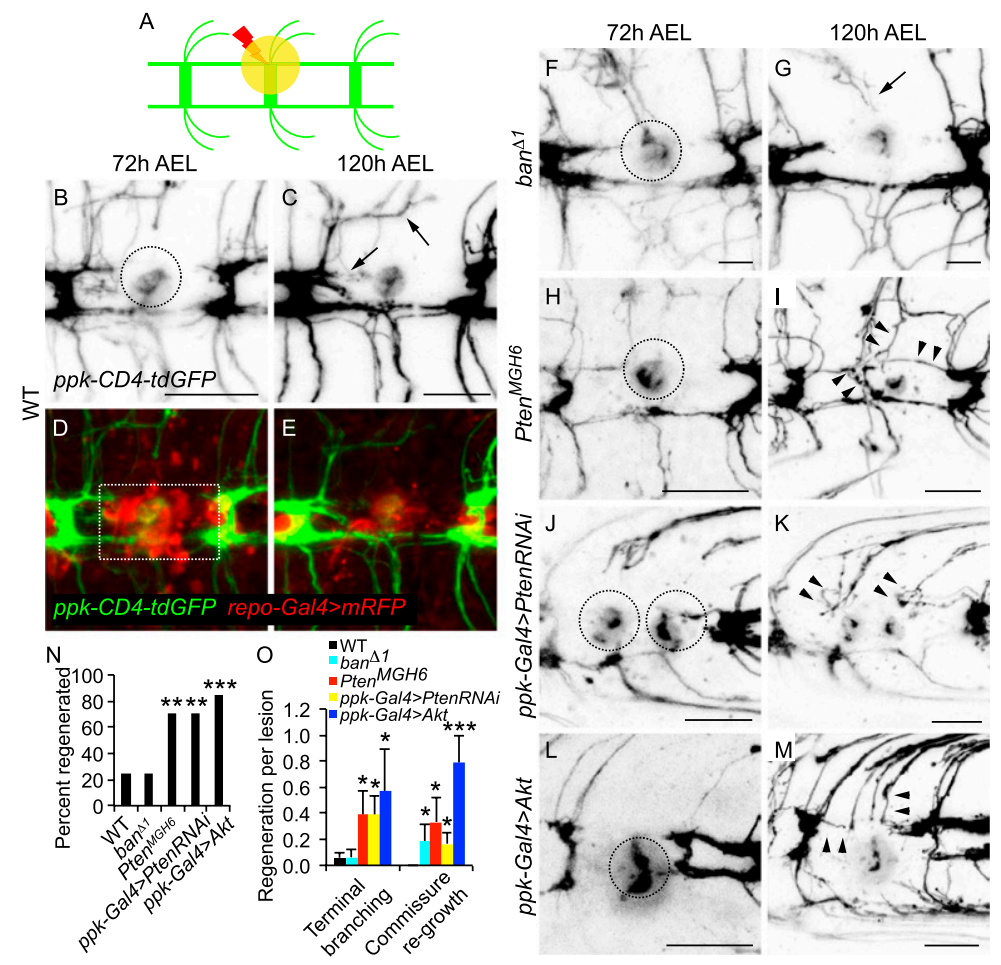
$0.0005 . n=28,12,17,17$, and 13 segments for wild type,
ban $^{\Delta 1}{ }^{\text {, Pten }}{ }^{M G H 6}$, ppk-Gal4>PtenRNAi, and ppk-Gal4>Akt. (O) Terminal branching and commissure regrowth were also significantly increased. Student's $t$-test, $\left(^{\star}\right) P<0.05 ;\left(^{\star \star \star}\right) P<0.0005 . n=18,8,9,9$, and 7 experiments for different genotypes.

Figure 3. da neuron axons in the VNC display limited regeneration, and activation of the PTEN-Akt pathway enhances their regeneration. (A) Schematic diagram showing class IV da neuron axotomy in the VNC. $(B-E)$ Axon regeneration of class IV da neurons in wild type (ppk-CD4-tdGFP/+; repo-Gal4, UAS-mRFP/+). (B) Axon severing was evident, showing axon stems and branches that retracted (circle). (C) Axons showed limited spouting and stalled at the lesion site (arrows). $(D, E)$ Glia accumulation was observed (box). $(F, G)$ No obvious improvement of axon regeneration was observed in $b_{a n}{ }^{\Delta 1}$ mutants (ppk-CD4-tdGFP/+; ban ${ }^{\Delta 1}$ ) (arrow). (H,I) Axon regeneration was dramatically stimulated in Pten $^{\text {MGH }}$ mutants $\left(\right.$ Pten $^{M G H 6}$; ppk-CD4-tdGFP/+). Besides increased sprouting, many axons were seen to regenerate substantially to regrow beyond lesion sites, exhibit terminal branching, and bridge commissure segments (arrowheads). (J,K) Axon regeneration was similarly enhanced in class IV da neurons expressing PtenRNAi (ppk-CD4-tdGFP/UAS-PtenRNAi; ppk-Gal4/+) (arrowheads). $(L, M)$ Class IV da neurons overexpressing Akt also showed significantly enhanced axon regeneration (ppk-CD4-tdGFP/UAS-Akt; ppk-Gal4/+) (arrowheads). Bar, $20 \mu \mathrm{m}$. $(N, O)$ Quantification of axon regeneration in the VNC. $(N)$ The regeneration percentage is significantly higher in Pten ${ }^{\text {MGH6 }}$ mutants or class IV da neurons expressing PtenRNAi or overexpressing Akt. Fisher's exact test, $\left.\left(^{\star \star}\right) P=0.0047,0.0047 ;{ }^{\star \star \star}\right) P=$ 
confirm axon transection (Fig. 3B, circle) and at $120 \mathrm{~h}$ AEL to assess regeneration. We found that class IV da neuron axons exhibited limited regeneration, with most of the axons stalling at the lesion site and showing limited sprouting (Fig. 3C, arrows). The failure of class IV da neuron axons to regenerate in the CNS, despite their ability to regenerate in the periphery, is similar to what has been found for DRG neurons after spinal cord injury in mice, raising the possibility that the VNC injury model for da neurons may provide insight into the processes that limit axon regeneration in the CNS.

Moreover, glia accumulation was also observed at the lesion site (Fig. 3D, box). Because this bears some resemblance to the glial scar seen in mammals, we decided to examine this structure in more detail. First, we performed higher-resolution confocal imaging from various angles and found that cellular structures labeled with repo-Gal4>mRFP rather than debris are enriched at the injury site $24 \mathrm{~h}$ after lesion (Supplemental Fig. S4C,G). Second, an important feature of the glial scar is the elevated level of chondroitin sulfate proteoglycans (CSPGs), which contribute to the inhibition of axon growth (Silver and Miller 2004). Therefore, we searched for Drosophila homologs of CSPGs and found two: kon-tiki (homolog of NG2/CSPG4) and Ptp99A (homolog of phosphacan/ PTPRZ1). Since the serine residue, which carries the chondroitin sulfate modification, is not conserved in kon-tiki (Schnorrer et al. 2007), we focused our analyses on Ptp99A. We found that Ptp99A expression is up-regulated at the lesion site, as demonstrated by antibody staining. It colocalizes with glial cells and surrounds the injury site, forming a ring-like structure (Supplemental Fig. $\mathrm{S} 4 \mathrm{D}, \mathrm{E}, \mathrm{H}, \mathrm{I})$, similar to what has been reported for astroglial scars in mammals (McKeon et al. 1999; Buss et al. 2009). In conclusion, these results suggest a potential role for glia in axon regeneration in the fly CNS, but further investigations are warranted to confirm the glial scar-like structure and determine whether Ptp99A contributes to the lack of regeneration in the fly CNS.

To test whether Pten loss of function also affects class IV da neuron axon regeneration in the VNC, we performed axotomy in $\mathrm{Pten}^{\mathrm{MGH}}$ mutants and found that the severed axons displayed increased sprouting compared with wildtype control. Indeed, many axons regrew beyond the lesion site, exhibited extensive terminal branching once they regrew into the commissures and reached their presumptive target area, and bridged the severed commissure segments (Fig. 3I, arrowheads). Quantitative analyses further showed a dramatic increase of the percentage of segments showing regeneration, from $25 \%$ in wild-type control to $70.6 \%$, as well as a significant increase in regenerating axons with "terminal branching" and "commissure regrowth" (Fig. 3N,O; see the Materials and Methods).

We also examined axon regeneration in larvae with Pten specifically knocked down in class IV da neurons using Pten RNAi (Ramachandran et al. 2009). A similar enhancement of axon regeneration in the VNC was observed as in Pten mutants (Fig. 3K [arrowheads], N,O). These data indicate that, as in mammals, PTEN functions cell- autonomously in neurons to inhibit axon regeneration in the CNS.

Last, we examined whether overexpression of Akt in class IV da neurons, which is negatively regulated by PTEN, may also lead to enhancement of axon regeneration in the CNS. Indeed, enhancing the Akt pathway activity by reducing PTEN or by Akt overexpression resulted in a comparable improvement of axon regeneration (Fig. 3M [arrowheads], $\mathrm{N}, \mathrm{O}$ ).

To rule out the possibility that the enhanced axon regeneration in the VNC might be due to developmental overgrowth of axon terminals independent of injury, we examined VNC axon terminals of class IV da neurons in wild type, Pten ${ }^{M G H 6}$ mutants, and larvae in which Pten is knocked down or Akt is overexpressed cell-autonomously. The gross VNC patterning was comparable among all the manipulation groups (Supplemental Fig. S5A-D), and no obvious overgrowth was observed at the individual axon terminals, based on MARCM or single-clone analyses (Supplemental Fig. S5E-I).

Taken together, these results indicate that the PTENAkt pathway represents a critical intrinsic neuronal regulator of axon regeneration in the CNS. Evolutionarily conserved across vertebrate and invertebrate species, this pathway may be activated by removing PTEN or ectopic Akt expression in neurons to trigger axon regeneration in the CNS.

\section{da neuron dendrites also regenerate in a cell type-specific manner}

To determine whether dendrites are capable of regeneration and whether they share any mechanism with axon regeneration, we first analyzed class IV da neuron ddaC. Dendriotomy was performed at $48 \mathrm{~h}$ AEL with the lesion site restricted to the primary dendritic branch point (Fig. 4A). This is because at this developmental stage, the distal dendrites are still actively growing. Thus, cutting the proximal dendrites ensures that the new dendrites growing out from the severed stem are due to regeneration. Complete severance was confirmed at $72 \mathrm{~h}$ AEL (Fig. 4B, arrowhead), and regeneration was scored at $120 \mathrm{~h}$ AEL. There was robust dendrite regeneration of $\mathrm{ddaC}$, which sent out new branches from the severed stem to reoccupy much of the vacant space (Fig. 4C, arrowhead). This phenomenon was quantified by determining the "regeneration percentage" (defined as the percentage of dendrites showing regeneration), revealing that $49.4 \%$ of the severed dendrites successfully regenerated (Fig. 4M). In those instances of failed regeneration, the injured branches stalled or retracted, and neighboring branches of the same neuron took over the vacant space (Fig. 4F). We further quantified the regeneration following ablation by measuring the "increase of branch points" and found that, on average, $\sim 10$ new branch points were added within $2 \mathrm{~d}$ (Fig. 4N). Last, we measured the increase of the total dendrite length and found that it was $\sim 600 \mu \mathrm{m}$ for ddaC (Fig. 4O). These findings show that class IV da neurons regenerate their dendrites in an all-or-none fashion; an injured dendritic branch either initiates regeneration or 
completely fails to regenerate. This is consistent with the stochastic nature of regeneration (Ghosh-Roy and Chisholm 2010; Samara et al. 2010).

To ask whether all da neurons possess the capacity for dendrite regeneration, we next analyzed class I da neurons. In contrast to class IV da neurons, class I da neurons did not exhibit substantial regrowth of dendrites (Fig. 4H,I, arrow), whereas neighboring dendrites from the same neuron showed obvious growth to scale the dendrites with the increasing larva size (Fig. 4I). The regeneration percentage for class I da neurons is $8.3 \%$, with no addition of branch points (Fig. $4 \mathrm{M}, \mathrm{N}$ ), and the increase of the total dendrite length averaged $\sim 18 \mu \mathrm{m}$ (Fig. 4O), which can be accounted for largely by dendritic scaling. The inability of class I dendrites to regrow has been reported in a previous study (Stone et al. 2010). Class I da neurons establish their characteristic morphology by $24 \mathrm{~h}$ AEL. These dendrites undergo scaling during larval development when the larva size increases drastically. In contrast, arbors of class III da neurons establish and cover their territory in the same time frame as class IV da neurons. Therefore, we analyzed class III da neurons marked with 19-12-Gal4>UAS-mCD8-

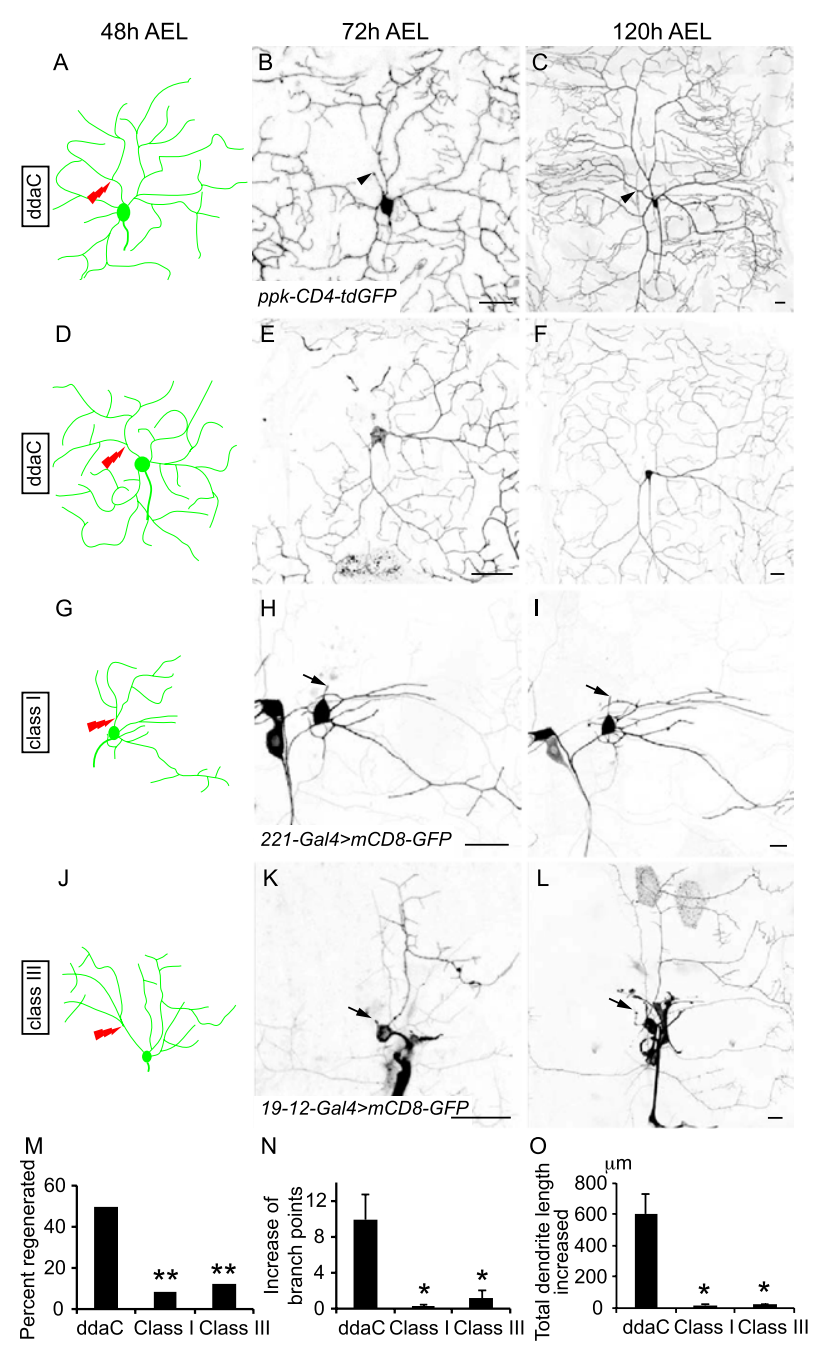

GFP and found their dendrites also failed to regenerate after injury, whereas neighboring dendrites from the same neuron grew and extended extensively (Fig. 4L [arrow], $\mathrm{M}-\mathrm{O})$. Together with the observations from axon regeneration, these results suggest that different classes of neurons differ in their ability to regenerate both dendrites and axons after lesion.

\section{da neuron dendrite regeneration is developmentally regulated}

The arbors of different da neurons are known to develop at different rates. Class III neurons cover their territory in the same time frame as class IV da neurons, first establishing receptive field coverage at $\sim 48 \mathrm{~h}$ AEL, followed by scaling and outgrowth of high-order branches, whereas class I da neurons covered their characteristic territory by $24 \mathrm{~h}$ AEL (Parrish et al. 2009). This raises the question of whether dendrite regeneration may be developmentally regulated. To explore this possibility, we performed dendriotomy at different developmental stages. For the class IV da neuron ddaC, we introduced lesion at $72 \mathrm{~h} \mathrm{AEL,}$ confirmed the transection at $96 \mathrm{~h} \mathrm{AEL} \mathrm{(Fig.} \mathrm{5A,} \mathrm{arrow),} \mathrm{and}$ scored dendrite regeneration at $120 \mathrm{~h}$ AEL (Fig. 5B, arrow). There was much less regeneration at this later stage; much of the vacant space remained unoccupied (Fig. 5B). Unlike the dendrite regeneration observed following lesion at $48 \mathrm{~h}$ AEL, the regeneration percentage was $20 \%$ instead of $49.4 \%$ and the "increase of branch points" averaged around one, rather than 10 (Fig. 5E,F). The increase of the total dendrite length was only $\sim 29 \mu \mathrm{m}$. Given that ddaC

Figure 4. da neuron dendrite regeneration displays cell type specificity. $(A-F)$ Dendrite regeneration of class IV da neuron ddaC, which is marked by ppk-CD4-tdGFP/+. $(A-C)$ Dendrites regenerated after lesion. (A) Dendriotomy was performed at $48 \mathrm{~h}$ AEL by targeting a two-photon laser to the primary branch point. (B) A complete transection was confirmed at $72 \mathrm{~h} \mathrm{AEL}$ (arrowhead). (C) Regeneration was scored at $120 \mathrm{~h} \mathrm{AEL}$, and $\mathrm{ddaC}$ dendrites showed substantial regrowth. New dendritic branches grew out from the severed stem and reoccupy the vacant space (arrowhead). (D-F) Dendrites failed to regenerate after lesion, with neighboring branches taking over the vacant space. $(G-I)$ Dendrite regeneration of class I da neurons, which is marked by 221-Gal4, UAS-mCD8-GFP. (G) The same dendriotomy protocol was performed as for ddaC. $(H)$ A complete transection was confirmed at $72 \mathrm{~h}$ AEL (arrow). (I) Regeneration was scored at $120 \mathrm{~h} \mathrm{AEL}$, and class I da neuron dendrites did not regenerate (arrow). ( $J-L$ ) Class III da neuron dendrites, which are marked by 19-12-Gal4, UAS-mCD8-GFP, also failed to regenerate after lesion. Bar, $20 \mu \mathrm{m}$. $(M-O)$ Quantification of dendrite regeneration. $(M)$ Regeneration percentages for class I and class III are significantly lower than that for ddaC. Fisher's exact test, $\left.{ }^{\star \star *}\right) P=0.0039,0.0032$. $(N)$ Increase of branch points is also significantly lower in class I and class III compared with ddaC. Student's $t$-test, $\left(^{\star}\right) P=0.028,0.026 . n=26,12$, and 16 for $\mathrm{ddaC}$, class I, and class III. (O) Total dendrite length increased is significantly lower in class I and class III compared with ddaC. Student's $t$-test, $\left(^{\star}\right) P<0.05 . n=26,12$, and 16 for ddaC, class I, and class III. 


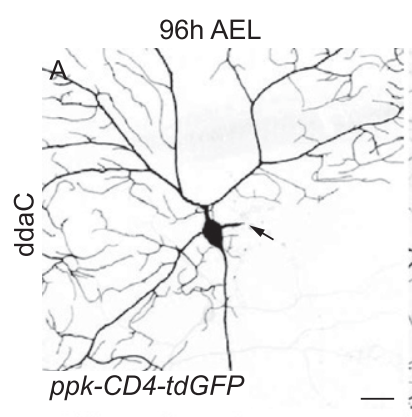

48h AEL
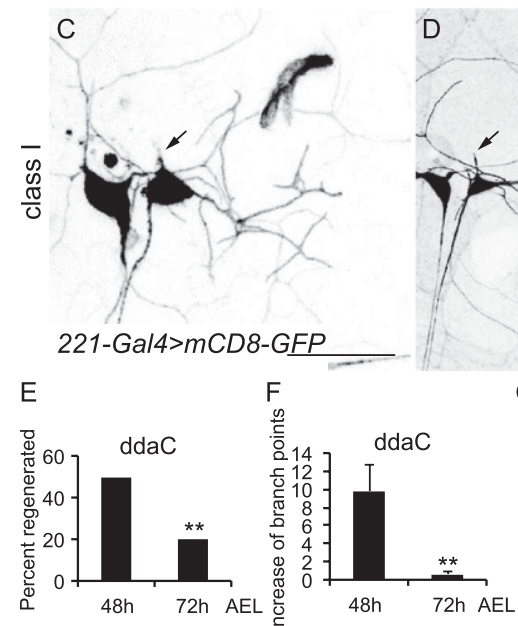

G
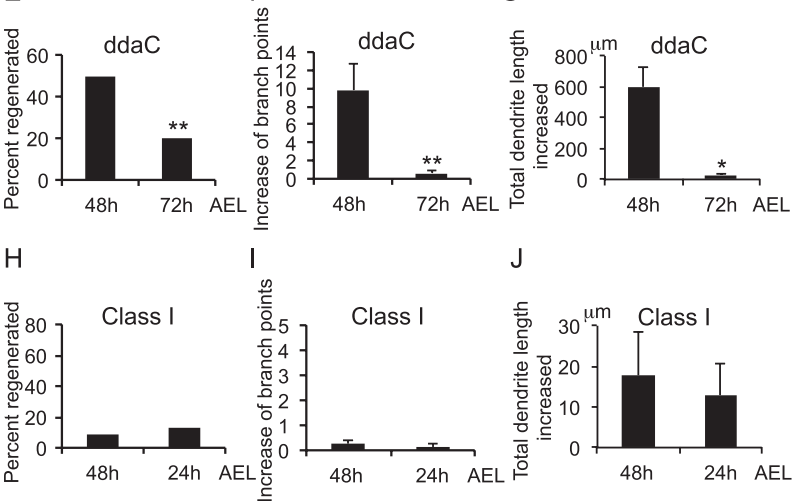

$\mathrm{J}$
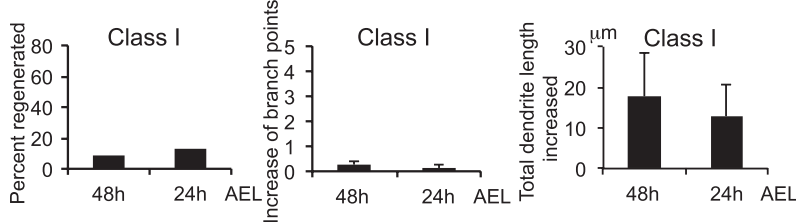

Figure 5. Developmental regulation of da neuron dendrite regeneration. $(A, B)$ Dendrite regeneration of class IV da neuron $\mathrm{ddaC}$, in which dendriotomy was performed at $72 \mathrm{~h}$ AEL and a complete transection was confirmed at $96 \mathrm{~h}$ AEL (arrow in $A$ ). (B) Regeneration was scored at $120 \mathrm{~h} \mathrm{AEL}$, and no obvious regeneration was observed, with most of the vacant space remaining unoccupied (arrow). ( $C, D)$ Dendrite regeneration of class I da neurons, in which dendriotomy was performed at $24 \mathrm{~h}$ AEL and a complete transection was confirmed at $48 \mathrm{~h} \mathrm{AEL} \mathrm{(arrow} \mathrm{in} \mathrm{C).}$ (D) Regeneration was scored at $120 \mathrm{~h} \mathrm{AEL}$, and still no obvious regeneration was observed (arrow). Bar, $20 \mu \mathrm{m}$. (E-G) Quantification of dendrite regeneration for ddaC. Dendrite regeneration of $\mathrm{ddaC}$ is down-regulated over time. $(E)$ The regeneration percentage for $\mathrm{ddaC}$ when lesioned at $72 \mathrm{~h}$ AEL is significantly lower than that when lesioned at $48 \mathrm{~h}$ AEL. Fisher's exact test, $\left({ }^{\star \star}\right) P=0.0042$. $(F)$ Increase of branch points is also significantly lower. Student's $t$-test, $\left(^{\star \star}\right) P=0.0026 . n=26$ and 25 for the two stages. $(G)$ Total dendrite length increased is significantly lower. Student's $t$-test, $\left(^{\star}\right) P=0.0059 . n=26$ and 25 for the two stages. $(H-I)$ Quantification of dendrite regeneration for class I. Dendrite regeneration of class I da neurons is not improved when the lesion was performed at $24 \mathrm{~h}$ AEL. The regeneration percentage, increase of branch points, and total dendrite length increased are not significantly different. $n=12$ and 8 for the two stages. dendrites were capable of substantial regeneration within $24 \mathrm{~h}$ following lesion at $48 \mathrm{~h}$ AEL (Fig. 6), the lack of regeneration at this later developmental stage indicates that dendrite regeneration is developmentally regulated, probably reflecting the onset of inhibitory mechanisms later in development (Parrish et al. 2009).

For class I da neurons, we sought to perform dendriotomy as early as possible to test whether we could capture a stage when class I dendrites might also show some degree of regeneration. To this end, we introduced lesion at $\sim 24 \mathrm{~h} \mathrm{AEL}$, immediately after hatching, which is the earliest time when we could isolate and sever individual primary dendrites. The transection was confirmed at $48 \mathrm{~h}$ AEL (Fig. 5C, arrow), and regeneration was scored at $120 \mathrm{~h}$ AEL (Fig. 5D, arrow). Even at this early stage, there was little indication for regeneration of class I da neuron dendrites, yielding a regeneration percentage of $12.5 \%$, no significant increase of branch points, and an increase of the total dendrite length by $\sim 13 \mu \mathrm{m}$ (Fig. $5 \mathrm{H}-\mathrm{J}$ ).

Taken together, these data suggest that da neuron dendrite regeneration displays cell type specificity and developmental regulation, with class IV but not class I or class III da neuron dendrites showing regeneration, while this capacity diminishes later in development.

\section{Dendrite regeneration is under the regulation} of the miRNA ban and the PTEN-Akt pathway

To begin searching for molecular pathways critical for dendrite regeneration, we focused our attention on miRNAs, given the involvement of miRNA-206 in the regeneration of neuromuscular synapses (Williams et al. 2009). The miRNA ban is a good candidate because a previous study has shown that it acts in epithelial cells to ensure that dendrite growth matches in scale with larval growth by suppressing Akt activity in da neurons (Parrish et al. 2009). To examine the class IV da neuron ddaC dendrite regeneration in $b_{a n}{ }^{\Delta 1}$ mutants, we performed dendriotomy at $48 \mathrm{~h} \mathrm{AEL}$, imaged the larvae $6 \mathrm{~h}$ after lesion to confirm transection, and monitored dendrite regeneration at $72 \mathrm{~h} \mathrm{AEL}$ and $120 \mathrm{~h}$ AEL. Interestingly, ban ${ }^{\Delta 1}$ mutants exhibited enhanced regeneration (Fig. 6D-F) when compared with wild-type control (Fig. 6M,N, black bar), with an $81.2 \%$ instead of $49.4 \%$ regeneration percentage and an increase of branch points of 38 instead of 13. The increase of the total dendrite length was also amplified to $\sim 1500 \mu \mathrm{m}$ (Fig. 6O, black bar). Interestingly, when we selectively analyzed the dendrites that showed regrowth, the addition of new branches in these regenerating dendrites, as measured by the increase of branch points and the increase of total dendrite length, appeared similar between wild-type control and $b n^{\Delta 1}$ mutants (Fig. 6N,O, white bars), suggesting that the enhanced regeneration is mainly due to the increased probability of regeneration. This mutant phenotype of enhanced regeneration was reduced by expressing ban in epithelial cells using 69B-Gal4 (Fig. 6M). Taken together with the non-cell-autonomous action of ban in epithelial cells to regulate dendritic scaling, these results indicate that ban signaling that originates in epithelial cells exerts an inhibitory effect on class 

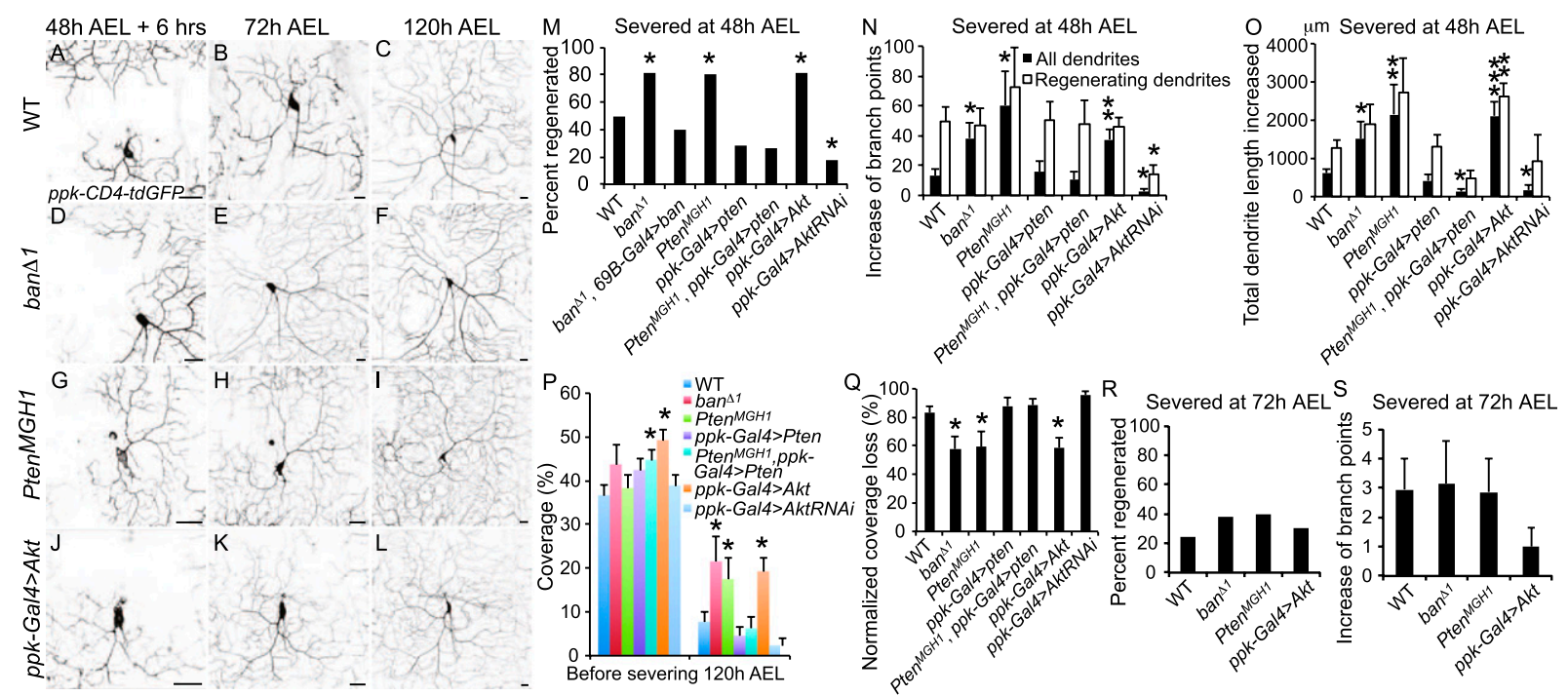

Figure 6. Dendrite regeneration is regulated by the Akt pathway involving ban and Pten. $(A-C)$ Dendrite regeneration of class IV da neuron $\mathrm{ddaC}$ in wild type (WT) (ppk-CD4-tdGFP/+). Dendriotomy was performed at $48 \mathrm{~h}$ AEL. $(A)$ A complete transection was confirmed $6 \mathrm{~h}$ after lesion. $(B, C)$ Regeneration was scored at $72 \mathrm{~h}$ AEL and $120 \mathrm{~h}$ AEL. $(D-F)$ Dendrite regeneration of ddaC in ban mutants is enhanced $\left(p p k-C D 4-t d G F P /+; b a n^{\Delta 1}\right)$. $(G-I)$ Dendrite regeneration of ddaC in Pten ${ }^{M G H 1}$ mutants is enhanced $\left(P t e n^{M G H 1}\right.$; ppk-CD4-tdGFP/+). (J-L) Dendrite regeneration of ddaC overexpressing Akt is similarly enhanced (ppk-CD4-tdGFP/UAS-Akt; ppkGal4/+). Bar, $20 \mu \mathrm{m}$. (M-O) Quantification of dendrite regeneration for ddaC in different genotypes. (M) The regeneration percentage for $\mathrm{ddaC}$ when lesioned at $48 \mathrm{~h}$ AEL is significantly increased in ${b a n^{\Delta 1}}^{\Delta}$ and Pten $^{M G H 1}$ mutants, as well as in neurons overexpressing Akt. Fisher's exact test, $\left(^{*}\right) P=0.0267,0.0219,0.0075$. The increase in $b a n^{\Delta 1}$ is eliminated by epithelial expression of ban (ppk-CD4-tdGFP/ UAS-ban; 69B-Gal4, ban $\left.{ }^{\Delta 1} / \mathrm{ban}^{\Delta 1}\right)$. The increase in Pten ${ }^{M G H 1}$ mutants is eliminated by neuronal expression of Pten (UAS-Pten, Pten $^{M G H 1} /$ Pten $^{M G H 1}$; ppk-Gal4/ppk-CD4-tdGFP). Knocking down Akt in da neurons using RNAi significantly decreased dendrite regeneration (ppk-CD4-tdGFP, UAS-Dcr-2/+; ppk-Gal4/UAS-AktRNAi). $n=79,16,20,20,28,19,22$, and 28 for wild type; ban ${ }^{\Delta 1}$; ban $^{\Delta 1}$, 69B-Gal4>ban; Pten ${ }^{M G H 1}$; ppk-Gal4>Pten; Pten ${ }^{M G H 1}$, ppk-Gal4>Pten; ppk-Gal4>Akt; and ppk-Gal4>AktRNAi. (N) Increase of branch points is also similarly increased in $b_{a n}{ }^{\Delta 1}$ and $P t e n^{M G H 1}$ mutants, as well as in neurons overexpressing Akt, when all of the severed dendrites were analyzed (black bars). Pten overexpression in da neurons overrides the increase in Pten ${ }^{\text {MGH1 }}$ mutants, while Akt knockdown in da neurons reduced regeneration. Student's $t$-test, $\left(^{*}\right) P<0.05$; $\left(^{\star \star}\right) P<0.005 . n=41,16,18,16,19,22$, and 27 for wild type; $b_{a n}{ }^{41}$; Pten ${ }^{M G H 1}$; ppk-Gal4>Pten; Pten ${ }^{M G H 1}$, ppk-Gal4>Pten; ppk-Gal4>Akt; and ppk-Gal4>AktRNAi. On the other hand, when only the dendrites that showed regeneration were selectively analyzed, no significant difference was observed (white bars), suggesting that once the dendrites regenerate, the regrowth speed itself is not dramatically altered. $n=11,13,15,5,4,18$, and 5 , respectively. $(O)$ Total dendrite length increased showed similar results. Student's $t$-test, $\left(^{\star}\right) P<0.05 ;\left(^{\star \star}\right) P<0.005 ;\left(^{\star \star \star}\right) P<0.0005 . n=57,16,18,16,19$, 22 , and 27 for wild type; ban $^{\Delta 1}{ }^{\text {; } P t e n}{ }^{M G H 1}$; ppk-Gal4>Pten; Pten ${ }^{M G H 1}$, ppk-Gal4>Pten; ppk-Gal4>Akt; and ppk-Gal4>AktRNAi when all the dendrites were analyzed, and $n=24,12,15,5,5,17$, and 5 when only the regenerating dendrites were analyzed. Because $b a n^{\Delta 1}, 69 B$ Gal4>ban larvae do not survive to $96 \mathrm{~h} \mathrm{AEL}$, only the regeneration percentage is presented. $(P, Q)$ Quantification of dendrite coverage for $\mathrm{ddaC}$ in different genotypes. $(P)$ The coverage of the dendritic branch was measured before severing and after regeneration at $120 \mathrm{~h}$ AEL. Whereas the coverage of the dendrite that was to be severed, as calculated by the ratio of the dendritic branch area divided by the entire dendritic field of the neuron, was comparable among the genotypes before severing, its coverage was significantly increased after regeneration in $\mathrm{ban}^{\Delta 1}{ }^{\text {, Pten }}{ }^{\mathrm{MGH1}}$ mutants, and Akt-overexpressing neurons compared with wild-type control. (Q) The coverage loss was calculated by measuring the decrease in the coverage of the injured dendritic branch before severing and at $120 \mathrm{~h} \mathrm{AEL}$, and this value was normalized to the initial coverage. A significant decrease of coverage loss was observed in ban ${ }^{\Delta 1}$, Pten ${ }^{M G H 1}$ mutants, and Aktoverexpressing neurons compared with wild-type control. Student's $t$-test, $\left({ }^{\star}\right) P<0.05 . n=30,16,19,15,19,21$, and 26 for wild type; ban $^{\Delta 1}{ }^{\text {; Pten }}{ }^{M G H 1}$; ppk-Gal4>Pten; Pten ${ }^{M G H 1}$, ppk-Gal4>Pten; ppk-Gal4>Akt; and ppk-Gal4>AktRNAi. (R,S) Quantification of dendrite regeneration for ddaC in different genotypes when dendriotomy was performed at $72 \mathrm{~h}$ AEL. In this paradigm, no significant difference was observed for regeneration percentage $(R)$ or increase of branch points $(S)$ among all of the manipulations. $n=40,13,15$, and 10 for wild type, ban $^{\Delta 1}$, Pten ${ }^{M G H 1}$, and ppk-Gal4>Akt.

IV da neuron dendrite regrowth after injury. As a result, the removal of ban significantly improves dendrite regeneration.

We next tested the involvement of PTEN, which acts upstream of Akt to suppress Akt activity. Removal of PTEN function in the Pten mutant Pten ${ }^{M G H 1}$ resulted in overgrowth of ddaC neuron dendrites (Supplemental Fig. S6E,F). This phenotype was much stronger than that of ban mutants, which only showed a slight increase of branching (Supplemental Fig. S6C,D). Similar phenotypes were also observed in Pten alleles Pten ${ }^{\text {MGH2 }}$ and Pten ${ }^{117}$. To test whether Pten functions cell-autonomously in ddaC neurons, we performed MARCM experiments and examined Pten ${ }^{M G H 1}$ mutant neuronal clones in an otherwise heterozygous background. We found that Pten ${ }^{\text {MGH1 }}$ mutant ddaC clones retained the exuberant overbranching phenotype (Supplemental Fig. S6G,H), indicating that PTEN function is required cell-autonomously in class IV 
da neurons to regulate dendrite patterning. We then examined PTEN function in dendrite regeneration and found that loss of PTEN function in Pten ${ }^{\text {MGH1 }}$ mutants enhanced dendrite regeneration (Fig. 6G-I,M-O, black bars), a phenotype similar to that of ban mutants. Moreover, as in $b{ }^{\Delta 1}$ mutants, the addition of new dendritic branches in the regenerating dendrites was not statistically different from wild-type control (Fig. 6N,O, white bars), suggesting that loss of PTEN function also enhances dendrite regeneration mainly by increasing the probability of initiating regrowth. Furthermore, we could reverse the enhanced dendrite regeneration phenotype by expressing PTEN in ddaC neurons using ppk-Gal4 (Fig. 6M-O). Thus, PTEN functions cell-autonomously to regulate dendrite regeneration.

We then examined the involvement of Akt, which is negatively regulated by both ban and Pten, in dendrite regeneration. We found that overexpression of Akt in ddaC neurons not only resulted in exuberant dendrite branching (Supplemental Fig. S6I,J), but also significantly enhanced dendrite regeneration (Fig. 6J-M,O, black bars)-again, largely by increasing the probability of regrowth, rather than altering the speed of new dendritic branch addition during regrowth (Fig. 6M,O, white bars). Conversely, knocking down Akt in ddaC with RNAi significantly reduced dendrite regeneration (Fig. 6M-O).

Given that ban and Pten loss of function as well as Akt overexpression resulted in increased dendrite branching and regeneration, we went on to determine whether they specifically up-regulate dendrite regeneration following injury. Each time a dendrite is severed, there are two competing processes: One is the regrowth of dendrites from the severed dendrites-what we define as regeneration —and the other is the overgrowth of the neighboring branches that invade the vacant area. If these manipulations simply result in general overgrowth, it should enhance both processes at the same time; i.e., both regeneration and overgrowth should be similarly enhanced, and therefore there would not be a dramatic change in the probability of these two processes. However, our results showed that in these manipulations, the probability of regeneration is dramatically enhanced. Furthermore, if these manipulations favored dendrite regeneration over general overgrowth, we would expect to see an expansion of the territory occupied by the regenerating branch in the manipulated larvae compared with wild-type control. To address this question, we compared the coverage of the dendritic branch before severing with that after regeneration at $120 \mathrm{~h}$ AEL. Whereas the coverage of the dendrite that was to be severed, as calculated by the ratio of the dendritic branch area divided by the entire dendritic field of that neuron, was comparable among all of the genotypes before severing (Fig. 6P), its coverage after regeneration was significantly increased by these experimental manipulations. While the regenerated dendrite took up $7.7 \%$ of the field in wild-type control, it covered up to $21.5 \%, 17.4 \%$, and $19.2 \%$ in ban $^{\Delta 1}$, Pten $^{M G H 1}$ mutants, and Akt-overexpressing neurons, respectively (Fig. 6P). Moreover, when we calculated the loss of coverage between the two stages normalized to the initial coverage, the coverage loss was significantly less in ban $^{\Delta 1}$, Pten ${ }^{M G H 1}$ mutants, and Akt-overexpressing neurons (Fig. 6Q). These results suggest that activation of the Akt pathway preferentially enhanced dendrite regeneration.

Last, given the improvement of class IV da neuron dendrite regeneration by activation of the Akt pathway, we went on to assess its effect on class I da neurons. Whereas their dendrite regeneration percentage showed a trend toward higher value in $b a n^{\Delta 1}$, Pten $^{M G H 1}$ mutants, and Akt-overexpressing neurons, there was no significant alteration in this measure or the increase of branch points (Supplemental Fig. S7), suggesting that activating the Akt pathway does not overcome the cell type barrier of dendrite regeneration.

In summary, activation of the Akt pathway results in improved dendrite regeneration. Together with the previous observations that ban signaling originates in epithelial cells (Parrish et al. 2009), whereas PTEN functions in da neurons, these findings reveal that both extrinsic and intrinsic forces work in concert to regulate dendrite regeneration.

Given that class IV da neurons gradually lose their capacity for dendrite regeneration during larval development, we tested whether activating the Akt pathway might sustain their regeneration potential when lesioned at $72 \mathrm{~h}$ AEL. We found no substantial dendrite regeneration at this late stage in ban, Pten mutants, or Aktoverexpressing da neurons (Fig. 6R,S; Supplemental Fig. S8). These observations suggest that activation of the Akt pathway alone is not sufficient to override the brake on dendrite regeneration later in development, raising the possibility that additional inhibitory mechanisms may be in place to limit the regrowth potential of da neuron dendrites later in development.

Having demonstrated that miRNA ban restrains dendrite regeneration through the Akt pathway in the peripheral nervous system, we went on to determine whether it also regulates axon regeneration in the CNS. We found that the ban loss of function did not result in enhanced axon regeneration in the VNC (Fig. 3F,G,N,O), indicating a specific involvement of ban in dendrite regeneration.

\section{Regenerating dendrites display mixed microtubule polarity}

Because we found that axon regeneration is accompanied by the reversal of microtubule polarity, we wondered whether a similar phenomenon occurs during dendrite regeneration. We first analyzed cases in which the severed dendrite failed to regrow and found that mixed EB1 polarity was evident in the neighboring branch that eventually took over the vacant space (Fig. 7A,C). As to those injured dendrites that initiated regeneration, there was a reversal of EB1 polarity in the regrowing dendrites but not in the neighboring branches (Fig. 7B,C; Supplemental Fig. S9A-C). This is in drastic contrast to class I da neurons, which do not regenerate their dendrites and do not show polarity reversal after dendrite lesion (Fig. 7D; Supplemental Fig. S9D,E; Stone et al. 2010). In summary, in class IV da neurons, a reversal of microtubule polarity is 
A

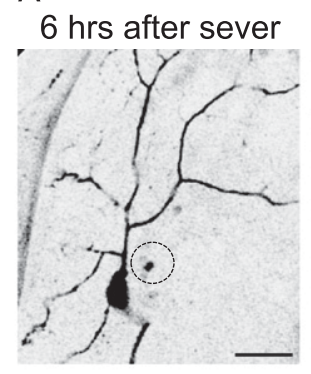

B

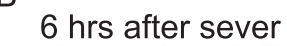

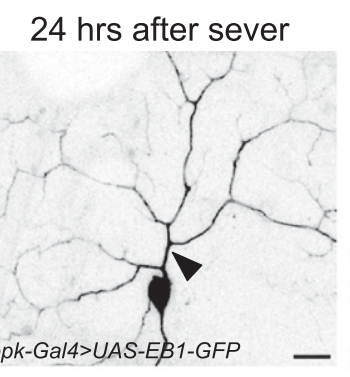

24 hrs after sever

\section{4 hrs after sever}

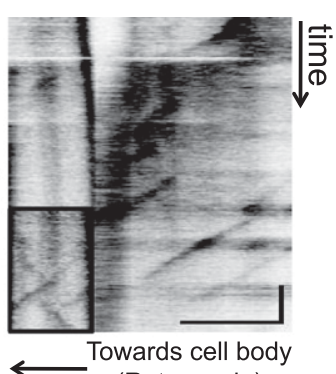

(Retrograde)
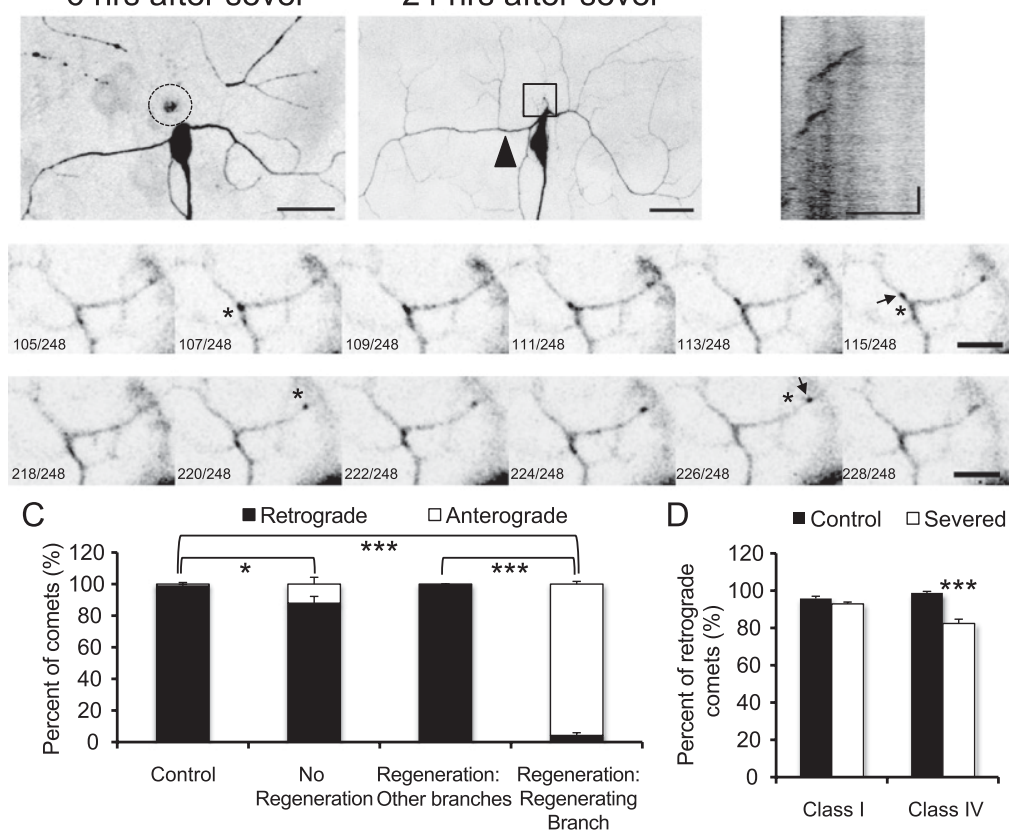

D

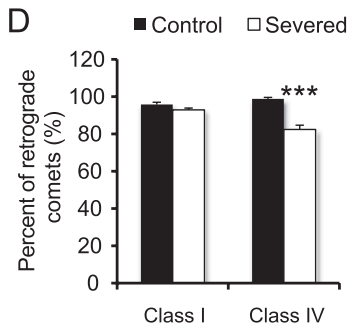

Figure 7. Different patterns of microtubule orientation result after dendriotomy in class IV da neurons, depending on regeneration. (A) Class IV da neurons that do not exhibit dendrite regeneration display an increase in anterograde EB1-GFP comets in neighboring branches. The boxed area of the kymograph shows a higher-contrast image of that section to show the mixed comet directionality. The arrowhead points to the branch used for generation of the kymograph. Bars: neuron images, $20 \mu \mathrm{m} ; X$-axis in kymographs, $5 \mu \mathrm{m}$; Y-axis in kymographs, $30 \mathrm{sec}$. (B) Class IV da neurons that do exhibit dendrite regeneration exhibit predominantly anterograde EB1GFP comets in the regenerating dendritic branch. The other branches do not exhibit mixed microtubule orientation. The arrowhead points to the branch used for generation of the kymograph. Dendrites were severed at $48 \mathrm{~h}$ AEL at the location represented by the dashed circle, and EB1 movies were captured at $72 \mathrm{~h}$ AEL. Panels from movies acquired of the boxed region show EB1-GFP comets moving in the anterograde direction. The asterisk indicates initial comet appearance, and the arrow indicates final comet position. Bars: neuron images, $20 \mu \mathrm{m}$; $X$-axis in kymographs, $5 \mu \mathrm{m}$; Y-axis in kymographs, $30 \mathrm{sec}$; montages, $3 \mu \mathrm{m}$. (C) Quantification of the percent of EB1 comets that grew in the retrograde direction in control versus severed class IV da neurons that did or did not regenerate. $(D)$ Comparison of the percent of EB1 comets that grew in the retrograde direction in control and severed class I versus class IV da neurons. Student's $t$-test, $\left.\left({ }^{\star}\right) P<0.05 ;{ }^{\star \star \star \star}\right) P<0.0005 . n>6$ neurons, and $n>100$ EB1 comets analyzed per condition. associated with the dendrites that possess the growing potential, whether it is a regenerating branch or an invading branch.

\section{Discussion}

In the present study, we showed that Drosophila sensory neuron dendrites and axons are capable of regeneration in a cell type-specific manner. While dendrites and axons share the same cell type specificity in their capacity for regeneration, they differ in their developmental regulation, with axons but not dendrites retaining the regeneration ability throughout larval development. We further show that the evolutionarily conserved PTEN-Akt signaling pathway is important for the regeneration of dendrites as well as axons and that both axon regeneration and dendrite regeneration are accompanied by the reversal of microtubule polarity.

\section{Axon scaling versus regeneration}

It is known that Drosophila larval axons undergo a scaling process in which axons substantially increase their length in accordance with the growth of the organism. Thus, it raises an important issue of whether larval axons regenerate or simply scale after axotomy. This question may be addressed with the following two considerations. First, larval axons scale while maintaining their neural connections; da neuron axons have already formed synaptic connections with neurons in the VNC, and these axon projections are not significantly altered as larvae grow in size (Kuo et al. 2005). Therefore, this increase of axon length does not involve bona fide axon pathfinding or synaptogenesis. Thus, axon scaling differs from the developmental axon outgrowth before synapse formation and is different from axon regeneration, which involves reinitiation of the developmental program for severed axons to generate growth cones or growth cone-like structures and pathfind to reach their targets. In the larval injury model, the axon is severed and therefore develops a new growing tip, reroutes following the presumptive trajectory by active or passive cues, and may or may not eventually establish synaptic contacts with their right targets in the CNS. This process resembles the regeneration program rather than axon scaling. Second, all of the da neurons, including class I, class III, and class IV, show similar axon scaling during larval stages. However, class IV but not class I or class III da neurons displayed axon 
regrowth after axotomy. The fact that only class IV da neurons are capable of regrowth, although all of these different types of da neurons undergo scaling, strongly suggests that class IV da neurons possess a unique regrowth potential that allows their severed axons to reinitiate the developmental program for axon outgrowth. For these reasons, we believe that a subset of the larval axons can regenerate after injury, although we cannot exclude the possibility that the ability of these axons to scale contributes to their regeneration potential. While this regeneration process may or may not fully recapitulate the regeneration program in adults, understanding how this process takes place in larvae will provide invaluable insights into the axon regeneration machinery.

What may determine the cell type specificity of axon and dendrite regeneration?

The ability of class IV but not class I or class III da neurons to readily regenerate their axons and dendrites could conceivably reflect cell type-specific features, including the transcription programs. One interesting question is whether the same program that governs the cell type morphology may also influence their regeneration capacity. For dendrite regeneration of class IV da neurons, either the regenerated dendrite or the neighboring dendritic branch continues to grow to fill the available space. Thus, they may possess a persistent growing potential that is inhibited by neighboring branches nearby so that the branches might overgrow if those inhibitory signals are removed. Therefore, with some branches removed due to injury, the remaining branches will regrow to take over the vacant space. Since class I and class III da neuron dendrites show very limited space-filling ability, these dendrites may lack the growth potential required for regeneration.

In response to injury, class IV da neurons regenerate their axons substantially, while class I da neurons partially reverse the microtubule polarity of nearby dendrites and convert one of these dendrites into a pseudoaxon (Stone et al. 2010). Taken together with our finding that class IV but not class I or class III da neurons are able to regenerate their dendrites, which are also associated with the reversal of microtubule polarity, these observations raise the question of whether pathways controlling neuronal polarity and/or cytoskeletal rearrangement may influence dendrite and axon regeneration.

\section{A combination of extrinsic and intrinsic factors determines dendrite regeneration}

Several lines of evidence suggest that dendrite regeneration depends on a balance of influences. First, there may be competition between de novo dendrite regeneration and invasion of neighboring branches. Successful regeneration prevents invasion and vice versa. Second, there could be a balance of extrinsic inhibitory cues, as in the form of the ban miRNA in epithelial cells, and intrinsic growth-promoting signals, as conveyed by the activation of the Akt pathway.

Moreover, given that activation of the Akt pathway at later stages of development is not sufficient to elevate the extent of dendrite regeneration to that during early larval development, it seems likely that either factors downstream from Akt are developmentally regulated to turn off the regeneration program at later stages or, alternatively, other pathways may contribute to this inhibition.

\section{A conserved growth program that regulates dendrite and axon regeneration}

Whereas dendrite and axon regeneration display differences with respect to developmental regulation, the PTEN-Akt pathway is important for regeneration of axons as well as dendrites. This pathway not only regulates the extent of dendrite growth of class IV da neurons during development, but also affects their dendrite regeneration and axon regeneration in the CNS. Together with previous work (Park et al. 2008; Liu et al. 2010), our results support the notion that modulating neuronal intrinsic PTEN and Akt activity is a potential therapeutic strategy for promoting axon regeneration and functional repair after CNS trauma.

A new injury model for systematic dissection of the machinery underlying dendrite regeneration and axon regeneration in the CNS

Our work focuses on class IV da neurons, which behave very differently from class I da neurons in regeneration. In particular, unlike class I da neurons, the class IV da neuron is capable of regenerating its axon in the periphery but not inside the CNS, thereby providing the first example of this phenomenon in invertebrates. A recent study of Caenorhabditis elegans PLM neurons, a type of mechanosensory neurons that consistently regrow their axons upon lasermediated axotomy, has identified multiple genes important for axon regeneration (Chen et al. 2011), illustrating the power of the genetic approach. The injury model involving Drosophila class IV da neuron axotomy in the CNS (VNC) in our study has the additional feature that it resembles the injury model involving mammalian DRG neuron axotomy in the CNS (spinal cord) at the cellular and molecular level: Both da neuron and DRG neuron axons regenerate poorly in the CNS even though they display robust regeneration in the periphery, and in both cases, axon regeneration in the CNS is enhanced by activation of the PTEN-Akt pathway. Importantly, while the PTEN-Akt pathway has been shown to be critical for mammalian axon regeneration in the CNS, this has not been shown in invertebrate models; for example, in C. elegans, PTEN (DAF-18) has no effect on axon regrowth (Chen and Chisholm 2011; Chen et al. 2011). Our finding underscores the usefulness of the Drosophila system that we developed as a model to uncover evolutionarily conserved mechanisms for CNS axon regeneration. Moreover, the elaborate and stereotyped dendritic branching pattern of da neurons provides a sensitive assay system to begin studying the injury responses and regeneration of dendrites, which may yield clues to facilitate studies of mammalian neuronal dendrites and identify novel approaches to promote dendrite recovery for the treatment of nervous system trauma. 


\section{Materials and methods}

\section{Immunohistochemistry and dendrite analyses}

Third instar larvae were fixed according to standard protocols. The following antibodies were used: rabbit anti- $\beta$ Gal (1:5000, Cappel), mouse anti-Ptp99A, (1:100, Developmental Studies Hybridoma Bank), and fluorescence-conjugated secondary antibodies (1:500, Jackson ImmunoResearch). Dendrite length analysis was performed using Imaris (Bitplane).

\section{Dendrite and axon lesion}

A single larva was mounted, and the dendrites or axons of a class IV, class I, or class III da neuron or the axon bundle of class IV da neurons in the VNC was targeted using a focused 930-nm twophoton laser ( 350-700 $\mathrm{mW}$ ) mounted on a custom-built Zeiss fluorescence microscope. Following lesion, animals were recovered to grape juice agar plates and imaged live at the appropriate stages. For dendrites, we chose to sever only the primary dendrites by targeting the laser to the primary dendritic branch point. For axons, we chose to sever the axon $\sim 20-50 \mu \mathrm{m}$ away from the cell body to minimize damage to the cell body. For axon bundle in the VNC, we ablated an area of $\sim 20 \mu \mathrm{m}$ in diameter at the commissure junction and only analyzed the segments where transected proximal axons can be clearly discerned when imaged $24 \mathrm{~h}$ after lesion.

\section{Quantitative analyses}

For dendrite regeneration, we used three parameters: regeneration percentage, which depicts the percentage of da neurons that showed regeneration among all of the da neurons that were severed; increase of branch points, which counts the addition of new dendritic branch points after severing; and total dendrite length increased. A regenerating dendrite is defined only if it showed increase in length and branch points. For axon regeneration, we used regeneration percentage, which depicts the number of regenerating axons among all the axons that were severed, and regeneration index, which is calculated as an increase of axon length/distance between the cell body and the axon converging point (DCAC) (Supplemental Fig. S1J-L). Using this value, the regeneration of $\mathrm{ddaC}$, class I, and class III da neurons can be compared directly because DCAC is largely similar for them in the same segment. In order to compare this value with v'ada, we first calculated a correction factor, which is the ratio of the DCAC for v'ada and that for ddaC. Thus, the regeneration index for ddaC and class I is normalized to that of v'ada by multiplying the correction factor. A regeneration index of 0 means no regeneration beyond the lesion site, with positive values representing regeneration and negative values representing retraction. A regenerating axon is defined only if it showed obvious regrowth beyond the lesion site. For axon regeneration in the VNC, we used regeneration percentage, which depicts the number of regenerating commissure segments among all of the segments that were severed; terminal branching, which counts the number of axons that regenerated, reached the commissure bundle, and appeared to form connections; and commissure regrowth, which counts the number of axons that had regenerated to bridge the boundaries of commissure segments longitudinally or laterally. A commissure segment is defined as regenerating when axons within that segment display clear regrowth.

\section{Acknowledgments}

We thank Susan Younger for help with genetics; Iswar Hariharan, Ernst Hafen, Stephan Cohen, Jay Parrish, Peter Soba, Rebecca
Yang, Yang Xiang, Quan Yuan, and Ye He for fly stocks; and Xi Huang, Yang Xiang, and members of the Jan laboratory for helpful discussions. This work was supported by post-doctoral fellowships from the Jane Coffin Childs Memorial Fund (to C.H. and to K.M.O.-M.) and by National Institutes of Health grant 2R37NS040929 (to Y.N.J.). This project was supported by the University of California through a continuation of the Roman Reed Spinal Cord Injury Research Fund of California. L.Y.J. and Y.N.J. are investigators of the Howard Hughes Medical Institute.

\section{References}

Awasaki T, Lai SL, Ito K, Lee T. 2008. Organization and postembryonic development of glial cells in the adult central brain of Drosophila. J Neurosci 28: 13742-13753.

Ayaz D, Leyssen M, Koch M, Yan J, Srahna M, Sheeba V, Fogle KJ, Holmes TC, Hassan BA. 2008. Axonal injury and regeneration in the adult brain of Drosophila. I Neurosci 28: 6010-6021.

Buss A, Pech K, Kakulas BA, Martin D, Schoenen J, Noth J, Brook GA. 2009. NG2 and phosphacan are present in the astroglial scar after human traumatic spinal cord injury. BMC Neurol 9: 32. doi: 10.1186/1471-2377-9-32.

Case LC, Tessier-Lavigne M. 2005. Regeneration of the adult central nervous system. Curr Biol 15: R749-R753. doi: 10.1016/ j.cub.2005.09.008.

Chen L, Chisholm AD. 2011. Axon regeneration mechanisms: Insights from C. elegans. Trends Cell Biol 21: 577-584.

Chen L, Wang Z, Ghosh-Roy A, Hubert T, Yan D, O'Rourke S, Bowerman B, Wu Z, Jin Y, Chisholm AD. 2011. Axon regeneration pathways identified by systematic genetic screening in C. elegans. Neuron 71: 1043-1057.

Clark IE, Jan LY, Jan YN. 1997. Reciprocal localization of Nod and kinesin fusion proteins indicates microtubule polarity in the Drosophila oocyte, epithelium, neuron and muscle. Development 124: 461-470.

Filbin MT. 2006. Recapitulate development to promote axonal regeneration: Good or bad approach? Philos Trans $R$ Soc Lond B Biol Sci 361: 1565-1574.

Fitch MT, Silver J. 2008. CNS injury, glial scars, and inflammation: Inhibitory extracellular matrices and regeneration failure. Exp Neurol 209: 294-301.

Gao FB, Brenman JE, Jan LY, Jan YN. 1999. Genes regulating dendritic outgrowth, branching, and routing in Drosophila. Genes \& Dev 13: 2549-2561.

Ghosh-Roy A, Chisholm AD. 2010. Caenorhabditis elegans: A new model organism for studies of axon regeneration. Dev Dyn 239: 1460-1464.

Goldberg JL, Klassen MP, Hua Y, Barres BA. 2002. Amacrinesignaled loss of intrinsic axon growth ability by retinal ganglion cells. Science 296: 1860-1864.

Grueber WB, Jan LY, Jan YN. 2002. Tiling of the Drosophila epidermis by multidendritic sensory neurons. Development 129: 2867-2878.

Hammarlund M, Nix P, Hauth L, Jorgensen EM, Bastiani M. 2009. Axon regeneration requires a conserved MAP kinase pathway. Science 323: 802-806.

Harel NY, Strittmatter SM. 2006. Can regenerating axons recapitulate developmental guidance during recovery from spinal cord injury? Nat Rev Neurosci 7: 603-616.

Kuo CT, Jan LY, Jan YN. 2005. Dendrite-specific remodeling of Drosophila sensory neurons requires matrix metalloproteases, ubiquitin-proteasome, and ecdysone signaling. Proc Natl Acad Sci 102: 15230-15235.

Kuo CT, Zhu S, Younger S, Jan LY, Jan YN. 2006. Identification of E2/E3 ubiquitinating enzymes and caspase activity regu- 
lating Drosophila sensory neuron dendrite pruning. Neuron 51: 283-290.

Lessing D, Bonini NM. 2009. Maintaining the brain: Insight into human neurodegeneration from Drosophila melanogaster mutants. Nat Rev Genet 10: 359-370.

Liu K, Lu Y, Lee JK, Samara R, Willenberg R, Sears-Kraxberger I, Tedeschi A, Park KK, Jin D, Cai B, et al. 2010. PTEN deletion enhances the regenerative ability of adult corticospinal neurons. Nat Neurosci 13: 1075-1081.

Lu B, Vogel H. 2009. Drosophila models of neurodegenerative diseases. Annu Rev Pathol 4: 315-342.

Marsh JL, Thompson LM. 2006. Drosophila in the study of neurodegenerative disease. Neuron 52: 169-178.

McKeon RJ, Jurynec MJ, Buck CR. 1999. The chondroitin sulfate proteoglycans neurocan and phosphacan are expressed by reactive astrocytes in the chronic CNS glial scar. I Neurosci 19: 10778-10788.

Moore DL, Blackmore MG, Hu Y, Kaestner KH, Bixby JL, Lemmon VP, Goldberg JL. 2009. KLF family members regulate intrinsic axon regeneration ability. Science 326: 298-301.

Park KK, Liu K, Hu Y, Smith PD, Wang C, Cai B, Xu B, Connolly L, Kramvis I, Sahin M, et al. 2008. Promoting axon regeneration in the adult CNS by modulation of the PTEN/ mTOR pathway. Science 322: 963-966.

Parrish JZ, Emoto K, Kim MD, Jan YN. 2007. Mechanisms that regulate establishment, maintenance, and remodeling of dendritic fields. Annu Rev Neurosci 30: 399-423.

Parrish JZ, Xu P, Kim CC, Jan LY, Jan YN. 2009. The microRNA bantam functions in epithelial cells to regulate scaling growth of dendrite arbors in drosophila sensory neurons. Neuron 63: 788-802.

Ramachandran P, Barria R, Ashley J, Budnik V. 2009. A critical step for postsynaptic F-actin organization: Regulation of Baz/ Par-3 localization by aPKC and PTEN. Dev Neurobiol 69: 583-602.

Ramon y Cajal S. 1928. Degeneration and regeneration of the nervous system. Oxford University Press, London.

Samara C, Rohde CB, Gilleland CL, Norton S, Haggarty SJ, Yanik MF. 2010. Large-scale in vivo femtosecond laser neurosurgery screen reveals small-molecule enhancer of regeneration. Proc Natl Acad Sci 107: 18342-18347.

Schnorrer F, Kalchhauser I, Dickson BJ. 2007. The transmembrane protein Kon-tiki couples to Dgrip to mediate myotube targeting in Drosophila. Dev Cell 12: 751-766.

Schwab ME, Bartholdi D. 1996. Degeneration and regeneration of axons in the lesioned spinal cord. Physiol Rev 76: 319-370.

Silver J, Miller JH. 2004. Regeneration beyond the glial scar. Nat Rev Neurosci 5: 146-156.

Stone MC, Nguyen MM, Tao J, Allender DL, Rolls MM. 2010. Global up-regulation of microtubule dynamics and polarity reversal during regeneration of an axon from a dendrite. Mol Biol Cell 21: 767-777.

Sugimura K, Yamamoto M, Niwa R, Satoh D, Goto S, Taniguchi M, Hayashi S, Uemura T. 2003. Distinct developmental modes and lesion-induced reactions of dendrites of two classes of Drosophila sensory neurons. J Neurosci 23: 3752-3760.

Sun F, He Z. 2010. Neuronal intrinsic barriers for axon regeneration in the adult CNS. Curr Opin Neurobiol 20: 510518.

Williams DW, Truman JW. 2005. Cellular mechanisms of dendrite pruning in Drosophila: Insights from in vivo timelapse of remodeling dendritic arborizing sensory neurons. Development 132: 3631-3642.

Williams DW, Kondo S, Krzyzanowska A, Hiromi Y, Truman JW. 2006. Local caspase activity directs engulfment of dendrites during pruning. Nat Neurosci 9: 1234-1236.
Williams AH, Valdez G, Moresi V, Qi X, McAnally J, Elliott JL, Bassel-Duby R, Sanes JR, Olson EN. 2009. MicroRNA-206 delays ALS progression and promotes regeneration of neuromuscular synapses in mice. Science 326: 1549-1554.

Xiang Y, Yuan Q, Vogt N, Looger LL, Jan LY, Jan YN. 2010. Light-avoidance-mediating photoreceptors tile the Drosophila larval body wall. Nature 468: 921-926.

Yan D, Wu Z, Chisholm AD, Jin Y. 2009. The DLK-1 kinase promotes mRNA stability and local translation in C. elegans synapses and axon regeneration. Cell 138: 1005-1018.

Yiu G, He Z. 2006. Glial inhibition of CNS axon regeneration. Nat Rev Neurosci 7: 617-627.

Zheng Y, Wildonger J, Ye B, Zhang Y, Kita A, Younger SH, Zimmerman S, Jan LY, Jan YN. 2008. Dynein is required for polarized dendritic transport and uniform microtubule orientation in axons. Nat Cell Biol 10: 1172-1180. 


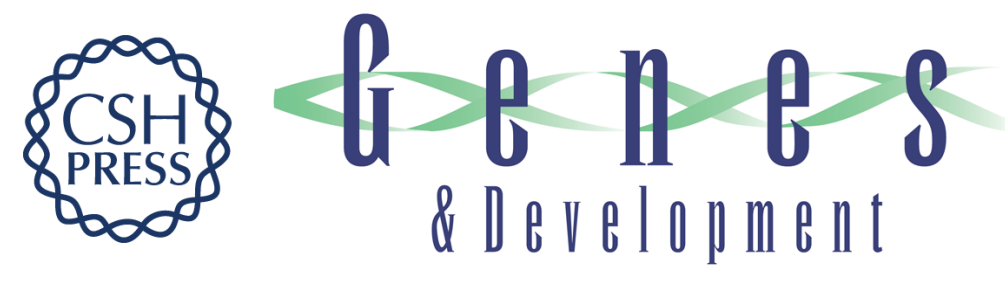

\section{Regeneration of Drosophila sensory neuron axons and dendrites is regulated by the Akt pathway involving Pten and microRNA bantam}

Yuanquan Song, Kassandra M. Ori-McKenney, Yi Zheng, et al.

Genes Dev. 2012, 26: originally published online July 3, 2012

Access the most recent version at doi:10.1101/gad.193243.112

\section{Supplemental http://genesdev.cshlp.org/content/suppl/2012/06/27/gad.193243.112.DC1 Material}

Related Content

No simpler than mammals: axon and dendrite regeneration in Drosophila Homaira Nawabi, Katherine Zukor and Zhigang He

Genes Dev. July , 2012 26: 1509-1514

References This article cites 41 articles, 15 of which can be accessed free at:

http://genesdev.cshlp.org/content/26/14/1612.full.html\#ref-list-1

Articles cited in:

http://genesdev.cshlp.org/content/26/14/1612.full.html\#related-urls

License

Email Alerting Receive free email alerts when new articles cite this article - sign up in the box at the top Service right corner of the article or click here.

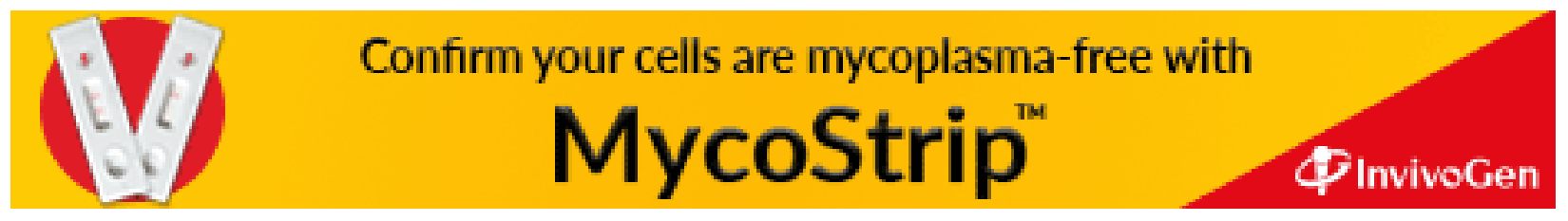

